\title{
Sensibilidad y especificidad del Test ScreeLing en usuarios afásicos en etapa aguda: un análisis preliminar
}

\author{
Valentina Mora-Castelletto a , Camila Hermosilla-Gotschlich a, Constanza Márquez-Espinoza a , \\ Sofía Puelma-Pistelli ${ }^{\mathbf{a}}$, Paulina Valdés-Flatow ${ }^{\mathbf{a}}$, Josette Sáez-Martínez ${ }^{\mathbf{b}}$, Bernardita Letelier-Vera ${ }^{\mathbf{b}}$, \\ Eduardo Fuentes-López a , Carolina Méndez-Orellana a,* \\ a Departamento Ciencias de la Salud, Pontificia Universidad Católica de Chile, Chile \\ b Servicio de Neurología, Complejo Asistencial Doctor Sótero del Rio, Servicio de Salud Metropolitano Sur Oriente, Chile
}

\section{RESUMEN}

En Chile, entre un $20 \%$ y un $38 \%$ de los usuarios que sufren un ataque cerebro-vascular (ACV) presentan afasia. Pese a su alta prevalencia no existen herramientas validadas que permitan realizar una caracterización lingüística temprana de la afasia. Por lo anterior, el objetivo del presente trabajo es analizar la sensibilidad y especificidad de la versión en español del ScreeLing en una muestra de usuarios afásicos después de producido un ACV. Se trata de un estudio transversal analítico en el cual se aplicó el test ScreeLing a 13 usuarios afásicos tras primer evento de ACV en etapa aguda ( $<6$ semanas tras ACV) y a 56 adultos neurológicamente sanos. Adicionalmente, se evaluó el nivel comprensivo y el lenguaje espontáneo de usuarios con afasia, con la versión abreviada del Token Test y Escala de Severidad de la Afasia (ASRS). Se construyeron modelos mediante regresiones logísticas, obteniendo sus respectivas áreas bajo la curva ROC. Los resultados muestran que el equilibrio entre sensibilidad y especificidad para el puntaje global del test ScreeLing se estableció en 67 puntos, obteniendo una sensibilidad del 94,6\% y especificidad del 100\%. El área bajo la curva ROC alcanzó 0,99 (IC 95\%: 0,97-1,00). En las subpruebas sintáctica y fonológica se alcanzó un puntaje de corte de 21 puntos, mientras que en la subprueba semántica se lograron 22 puntos. Se concluye que el ScreeLing en español posee una alta sensibilidad y especificidad para la detección de déficits lingüísticos post ACV similar a los resultados encontrados en la versión original del test en holandés. Esfuerzos futuros deben concentrarse en ampliar la muestra de usuarios afásicos con distintos perfiles de severidad.

\section{Sensitivity and Specificity of the Spanish version of the ScreeLing Test in aphasic patients at the acute phase: A pilot study}

\section{ABSTRACT}

In Chile, aphasia is present in $20 \%$ to $30 \%$ of stroke patients. Currently, speech and language therapists do not have a standardized test to characterize aphasic patients' linguistics disorders at the acute phase. The aim of this study iso analyze the sensibility and specificity of the Spanish version of the ScreeLing test in aphasic patient post-stroke at the acute stage. To do so, in a transversal study design, acute aphasic patients ( $<6$ weeks post-stroke) and healthy adults were evaluated with the Spanish version of the ScreeLing test. Additionally, language comprehension and spontaneous speech were assessed with the shortened version of the Token Test and the Aphasia Severity Rating Scale, respectively. We calculated the Area Under ROC Curve (AUC) to choose the cut-off that provided the best balance between sensibility and specificity. We included 13 aphasic patients and 56 healthy adults. The best balance between sensibility and specificity was found to be with 67 points, with a sensibility of $94,6 \%$ and specificity of $100 \%$. The AUC ROC was 0,99 (IC 95\%: 0,97-1,00). For the subtest measuring syntax and phonology, the cut-off score was 21 points, and for the semantic subtest, 22 points. In conclusion, the Spanish version of the ScreeLing test, similar to its original Dutch version, demonstrated high specificity and sensibility to detect linguistic deficits in acute aphasic patients post-stroke. Future efforts aim to increase the sample size of patients considering different language severity profiles.

*Autor/a correspondiente: Carolina Méndez

Email: carolinamendez@uc.cl

Recibido: 03-12-2019

Aceptado: 09-04-2020

Publicado: 23-11-2020 


\section{INTRODUCCIÓN}

En Chile, las enfermedades cerebrovasculares (ECV) son la primera causa de muerte, el año 2010 el 9\% de los decesos fue producto de ECV (Ministerio de Salud Chile, 2013). El Ataque Cerebrovascular (ACV) isquémico corresponde a la causa más frecuente de ECV, alcanzando un $65 \%$ de casos al año (Ministerio de Salud Chile, 2013). Una de las consecuencias con mayor impacto es la afasia, ya que entre un $20 \%$ y un $38 \%$ de los pacientes en etapa aguda post ACV la presenta. Además, con la edad aumenta su incidencia (González et al., 2017).

Dada la gran incidencia de la afasia, es muy importante contar con instrumentos de evaluación temprana que ayuden a generar un diagnóstico y orienten la intervención temprana (Flamand-Roze et al., 2011), maximizando así las ganancias de la rehabilitación (Salter et al., 2006). Considerando el reporte de Nouwens y colaboradores del año 2014, los pacientes se benefician de la terapia cognitiva lingüística durante los primeros 3 meses tras un ACV (Nouwens et al., 2014). Por ello caracterizar el perfil lingüístico y comunicativo de forma temprana es necesario para iniciar la terapia en la fase aguda. Así, la evaluación temprana se hace necesaria para obtener una línea de base y a partir de ella proyectar los resultados de la terapia y los progresos del paciente (Laska et al., 2007).

Los tests más utilizados para la evaluación del lenguaje, tales como el test de Boston para el diagnóstico de afasia, el test de Vocabulario de Boston y la Western Aphasia Battery (WAB), no son los más apropiados para aplicar durante la fase aguda de un $\mathrm{ACV}$. Lo anterior debido a que requieren mucho tiempo de aplicación e incluyen contenidos que entregan información que no contribuye con la intervención de la afasia durante esta etapa. A nivel global, las escalas utilizadas en etapas agudas, como es el caso del National Institute of Health Stroke Scale (NIHSS) (Adams et al., 1999), no detectan de manera confiable la presencia y severidad de afasia (Flamand-Roze et al., 2011). En estos momentos en Chile, no existe una herramienta adecuada que se pueda utilizar en etapas agudas post ACV.

La revisión sistemática desarrollada por El Hachioui et al. (2017) analiza las propiedades psicométricas de cuatro tests de screening para evaluar la presencia de afasia: Language Screening Test (LAST), Frenchay Aphasia Screening Test (FAST), Mississippi Aphasia Screening test (MAST) y el test ScreeLing. Esta revisión destaca el test ScreeLing, porque su proceso de validación es más exhaustivo que los otros tests. Ello debido a que incorpora una metodología de doble ciego (El Hachioui et al., 2017).
El test ScreeLing (El Hachioui et al., 2012) es una prueba de barrido que evalúa cuantitativamente los componentes fonológico, sintáctico y semántico. Entrega un perfil lingüístico del cuadro afásico que favorece la evaluación transterapéutica. Junto con esto, presenta características psicométricas que lo destacan como la herramienta óptima para la evaluación del lenguaje en etapa aguda post ACV (El Hachioui et al., 2012), siendo altamente específico (96\%) y sensible (86\%). Requiere corto tiempo de administración (aproximadamente 15 minutos) y puede ser aplicado en pacientes hospitalizados bedside (El Hachioui et al., 2012, 2017).

En Chile, la guía clínica Garantías Explícitas en Salud (GES) de ACV recomienda la evaluación y el inicio de la terapia fonoaudiológica de forma temprana (Ministerio de Salud Chile, 2013). Esta guía propone utilizar el Test de Boston como instrumento de evaluación. Sin embargo, el uso de este instrumento no es óptimo para evaluar pacientes hospitalizados, por su larga duración. Además, no está cultural ni lingüísticamente adaptado para la realidad chilena. Por ello, es necesario contar con instrumentos validados en nuestra población, que permitan una evaluación lingüística exhaustiva, con el objetivo de brindar una mejor recolección de información y encauzar la intervención fonoaudiológica.

El presente estudio tiene por objetivo analizar la sensibilidad y especificidad de la versión en español del test ScreeLing en una muestra de población chilena en etapa aguda post ACV. Para lograr el objetivo, se comparó las puntuaciones obtenidas por adultos neurológicamente sanos y usuarios con diagnóstico de afasia en etapa aguda post ACV. Dichas puntuaciones corresponden tanto al nivel global como a cada componente lingüístico del Test ScreeLing. Adicionalmente, se obtuvieron los puntajes de corte para cada componente lingüístico y para el puntaje global. Estos puntajes permitirán detectar la presencia o ausencia de afasia.

\section{MATERIALES Y MÉTODOS}

\section{Participantes}

Se seleccionó una muestra por conveniencia de pacientes en fase aguda posterior a un ACV, pertenecientes al servicio de Neurología y Neurocirugía del Centro Asistencial Sótero del Río (CASR) y del Centro de Salud UC Christus Marcoleta. Se incluyó a pacientes usuarios afásicos con las siguientes características: hablantes de español de Chile mayor de 18 años, cursando fase aguda ( $<6$ semanas posterior al ACV) después de un primer ACV 
isquémico o hemorrágico, candidatos a ser evaluados con versión abreviada del Token Test (De Renzi \& Faglioni, 1978). Los criterios de exclusión abarcaron alguna de las siguientes características: analfabetismo, discapacidad auditiva y/o visual severa, dislexia, disartria severa, diagnóstico de enfermedad psiquiátrica o neurodegenerativa, antecedentes de enfermedades neurológicas. De este modo, el grupo de casos correspondió a 13 sujetos ( 2 mujeres) entre 18 y 88 años de edad, con un promedio de 64 años $( \pm 17,96)$.

El grupo control también fue una muestra por conveniencia que se reclutó mediante dos procedimientos. Uno fue invitar a familiares de los pacientes y el otro fue convocar a participar del estudio, a través de comunicados expuestos en distintos centros médicos. Los criterios de exclusión fueron los mismos utilizados para conformar la muestra de pacientes afásicos. Así, este grupo se constituyó por 56 personas, ( 33 mujeres) entre 22 y 88 años de edad, con un promedio de 54,5 años $( \pm 15,23)$, que estaban neurológicamente sanos y eran hablantes de español de Chile.

Es destacable señalar, que existen diferencias significativas respecto al nivel educacional y de sexo en ambos grupos (Tabla $1)$.

Todos los participantes firmaron voluntariamente un consentimiento informado aprobado por el Comité Ético Científico del Servicio Metropolitano Sur Oriente y Comité Ético Científico de Facultad de Medicina, Pontificia Universidad Católica de Chile.

Tabla 1. Características demográficas y clínicas de los pacientes incluidos en el estudio.

\begin{tabular}{|c|c|c|c|}
\hline & Caso & Control & \\
\hline & $(\mathrm{n}=13)$ & $(\mathrm{n}=56)$ & Valor $\mathrm{P}$ \\
\hline Edad, años, promedio (DS) [rango] & $64( \pm 17,96)[18-88]$ & $54.5( \pm 15,23)[22-88]$ & $0,992^{(1)}$ \\
\hline Género, n (\%) & & & $0,011^{(2)}$ \\
\hline Femenino & $2(15,4)$ & $23(41,1)$ & \\
\hline Masculino & $11(84,6)$ & $33(58,9)$ & \\
\hline Lateralidad $(\mathrm{EHI})^{(4)}, \mathrm{n}(\%)$ & & & $0,322^{(2)}$ \\
\hline Diestro & $12(92,3)$ & $52(92,9)$ & \\
\hline Zurdo & $0(0)$ & $4(7,1)$ & \\
\hline Ambidiestro & $0(0)$ & $0(0)$ & \\
\hline Desconocido & $1(7.7)$ & $0(0)$ & \\
\hline Años de escolaridad, n (\%) & $10,6( \pm 3,8)$ & $13,4( \pm 3,5)$ & $0,017^{(3)}$ \\
\hline \multicolumn{4}{|l|}{ Tipo de ACV, n (\%) } \\
\hline Isquémico & $11(84.6)$ & \multirow{3}{*}{-} & \multirow{3}{*}{-} \\
\hline Hemorrágico & $1(7,7)$ & & \\
\hline Ambos (isquémica y hemorrágico) & $1(7,7)$ & & \\
\hline \multicolumn{4}{|l|}{ Localización del ACV, n (\%) } \\
\hline Izquierdo & $6(46,2)$ & & \\
\hline Derecho & $2(15,4)$ & - & - \\
\hline Ambas & $1(7,7)$ & & \\
\hline Desconocido & $4(30,8)$ & & \\
\hline
\end{tabular}

Abreviaciones: DS= Desviación estándar, $\mathrm{n}=$ número, ${ }^{(1)}$ Independent t-test, ${ }^{\left({ }^{2}\right)}$ Chi-squared test, ${ }^{(3)}$ Mann-Whitney test, ${ }^{(4)}$ Evaluación de la lateralidad de Edinburgo. 


\section{Instrumentos}

El Test ScreeLing evalúa cuantitativamente a través de 3 subpruebas los componentes fonológico, sintáctico y semántico del lenguaje. Cada subprueba cuenta de 24 ítems que son evaluados en modalidad expresiva y comprensiva. Los estímulos utilizados corresponden a imágenes y palabras y/o frases escritas. las palabras pueden ser leídas en caso de afasias moderadas a severas. Los puntajes de corte, descritos en Holanda para la puntuación global del test ScreeLing, fueron 68 puntos, con un máximo de 72. En el caso de las subpruebas se estableció en 22 puntos el puntaje de corte para cada componente, cuyo máximo fue 24 puntos (El Hachioui et al., 2012). La adaptación lingüística, cultural y la validación de contenido de este instrumento en español fue realizada por un equipo constituido por lingüistas clínicos (2 lingüistas nacionales y 2 internacionales) y fonoaudiólogos (12 profesionales nacionales con 10 años de experiencia en área de evaluación en adultos). Su versión final obtuvo un índice de validez de contenido (IVC) de Lawshe de 0,97 y fue piloteada en 30 sujetos sanos y 15 pacientes afásicos.

Adicionalmente, para poder caracterizar objetivamente las alteraciones a nivel de producción y comprensión de los pacientes afásicos se utilizó la versión abreviada del Token Test (De Renzi \& Faglioni, 1978). Además, se realizó un análisis clínico del lenguaje espontáneo, mediante la Escala de severidad y perfil de características del habla, propuesta en el Test de Boston para el diagnóstico de afasia (Goodglass et al., 2005).

\section{Procedimientos}

Los pacientes seleccionados fueron evaluados en la fase aguda, en un promedio de 17 días $( \pm 10)$ tras el evento ACV. El origen de $\mathrm{ACV}$ fue isquémico en 11 de los pacientes reclutados. El tipo y localización de las lesiones se muestra en tabla 1. (Datos de sitio de la lesión y puntajes de Token Test y ASRS son proporcionados en los datos suplementarios).

A su vez, los datos demográficos (tales como sexo, edad, escolaridad, dominancia manual) y clínicos (tipo de ACV, fecha del ACV) de los usuarios con afasia fueron recolectados a través de una anamnesis y ficha clínica de los establecimientos hospitalarios. La dominancia manual fue evaluada con el inventario de Edinburgo.

La evaluación mediante el Token Test y la versión al español del Test ScreeLing tuvo una duración aproximada de 30 minutos y fue realizada por estudiantes de pregrado en su penúltimo año de formación profesional, siendo supervisadas por un tutor. El Token Test se presentó de forma impresa y se utilizaron las fichas acrílicas de colores de $3 \mathrm{~cm}$ y el test ScreeLing se aplicó en Tablets (pantalla tamaño: 7,9" o 12.9").

\section{Análisis estadístico}

Se estimaron estadígrafos descriptivos como el promedio y desviación estándar para las variables continúas con distribución normal, mientras que mediana y rango intercuartílico para aquellas con distribución sesgada. Con el fin de conocer la distribución de las variables se aplicó la prueba de Shapiro-Wilk. En el caso de las variables categóricas se obtuvo frecuencias relativas y absolutas.

Se realizó el test de Spearman para estudiar la posible correlación entre la edad y los puntajes obtenidos por el grupo control y grupo caso. Dada la distribución sesgada de las puntuaciones en el test ScreeLing, se utilizó la prueba de Mann-Whitney para comparar las medianas entre ambos grupos. Mediante una regresión logística se estimaron los odds ratios (OR), los valores de sensibilidad y especificidad para cada uno de los puntajes obtenidos tanto en el puntaje global como en las puntuaciones de sus subpruebas. Los valores de sensibilidad y especificidad fueron graficados mediante las áreas bajo la curva ROC para el puntaje global y para el de las subpruebas.

En todas las pruebas estadísticas se consideró como significativo un alpha de 0.05. El análisis estadístico de los resultados se realizó en Stata versión 12.

\section{RESULTADOS}

La correlación entre la edad y tanto las puntuaciones globales como las de cada subprueba fue baja y en ningún caso significativa, para el grupo de casos y para los adultos neurológicamente sanos. Dado esto, no se realizó ajuste por esta variable en el modelo. En la Tabla 2 se observan los puntajes obtenidos por ambos grupos en el test ScreeLing, considerando sus subpruebas semántica, fonológica y sintáctica.

Debido a que tanto las puntuaciones globales como las de cada subprueba mostraron un comportamiento sesgado (no normal) se utilizó la prueba no paramétrica de Mann-Whitney al comparar el grupo de casos con los adultos neurológicamente sanos. Destacó que el grupo control obtuvo una puntuación significativamente mayor en el test ScreeLing, en comparación al grupo de casos $(p<0,001)$. Lo anterior también es válido para las subpruebas del nivel fonológico, sintáctico y semántico (Tabla 2). 
Tabla 2. Evaluación pre y post intervención según la etapa del DN.

\begin{tabular}{|c|c|c|c|c|c|c|}
\hline ScreeLing & Grupo & Mínimo & Máximo & Promedio y (DE) & IC $95 \%^{(a)}$ & $\begin{array}{c}\text { p-value para la } \\
\text { diferencia }^{(\mathrm{b})}\end{array}$ \\
\hline \multirow[t]{3}{*}{ Semántica } & Controles & 18 & 24 & $23,13( \pm 1.15)$ & $22,82-23,43$ & \\
\hline & & & & & & $<0,001$ \\
\hline & Casos & 5 & 22 & $17,31( \pm 4,84)$ & $14,38-20,23$ & \\
\hline \multirow[t]{3}{*}{ Fonológica } & Controles & 21 & 24 & $23,56( \pm 0,73)$ & $23,37-23,76$ & \\
\hline & & & & & & $<0,001$ \\
\hline & Casos & 5 & 24 & $17,04( \pm 6,72)$ & $12,98-21,10$ & \\
\hline \multirow[t]{3}{*}{ Sintáctica } & Controles & 15 & 24 & $23,29( \pm 1,41)$ & $22,91-23,66$ & \\
\hline & & & & & & $<0,001$ \\
\hline & Casos & 5 & 20 & $15,92( \pm 4,13)$ & $13,42-18,42$ & \\
\hline \multirow[t]{3}{*}{ Global } & Controles & 59 & 72 & $69,99( \pm 2,35)$ & $69,36-70,62$ & \\
\hline & & & & & & $<0,001$ \\
\hline & Casos & 16 & 65 & $50,27( \pm 14,5)$ & $41,50-59,03$ & \\
\hline
\end{tabular}

${ }^{(a)}$ Intervalo de confianza al 95\%. ${ }^{(b)} \mathrm{P}-$ value obtenido mediante la prueba no paramétrica de Mann-Whitney.

En la Tabla 3 se observan los resultados para el modelo de regresión logística en que se tuvo como variable dependiente al grupo (casos versus controles) y como predictor el puntaje global del test ScreeLing y sus subpruebas. En cuanto al puntaje global se obtuvo un OR de 0,51. Lo anterior significa que por cada punto de incremento en el test, disminuye cerca del 50\% la posibilidad de pertenecer al grupo de casos. Las diferencias en OR resultaron ser de menor magnitud para los otros componentes, aunque en todos los casos fueron estadísticamente significativas.
Tabla 3. Odds ratio (OR) para la relación entre el grupo (usuarios afásicos versus grupo control) y las puntuaciones del test ScreeLing y sus subpruebas $^{(1)}$.

\begin{tabular}{lccc}
\hline ScreeLing & OR & IC 95\% & $p$-value \\
\hline Subprueba semántica & 0,36 & $0,20-0,65$ & $<0,01$ \\
Subprueba fonológica & 0,37 & $0,19-0,72$ & $<0,01$ \\
Subprueba sintáctica & 0,34 & $0,19-0,62$ & $<0,001$ \\
Puntaje global & 0,51 & $0,34-0,77$ & $<0,01$ \\
\hline
\end{tabular}

${ }^{(1)}$ Se utiliza un modelo de regresión logística univariado obteniendo los odds ratio (OR). 
En la Figura 1 se observan las curvas ROC y sus respectivas áreas, las que permiten visualizar la sensibilidad y especificidad alcanzada con la versión español del ScreeLing. Para el caso del puntaje global, ésta alcanzó un valor de 0,99. Al considerar un puntaje de corte de 67 puntos, se tiene un equilibrio maximizando sensibilidad y especificidad, siendo $94,64 \%$ y $100 \%$, respectivamente (Tabla 3). Por lo tanto, obtener 67 o menos puntos indicaría la presencia de una alteración lingüística propia de la afasia en etapa aguda.
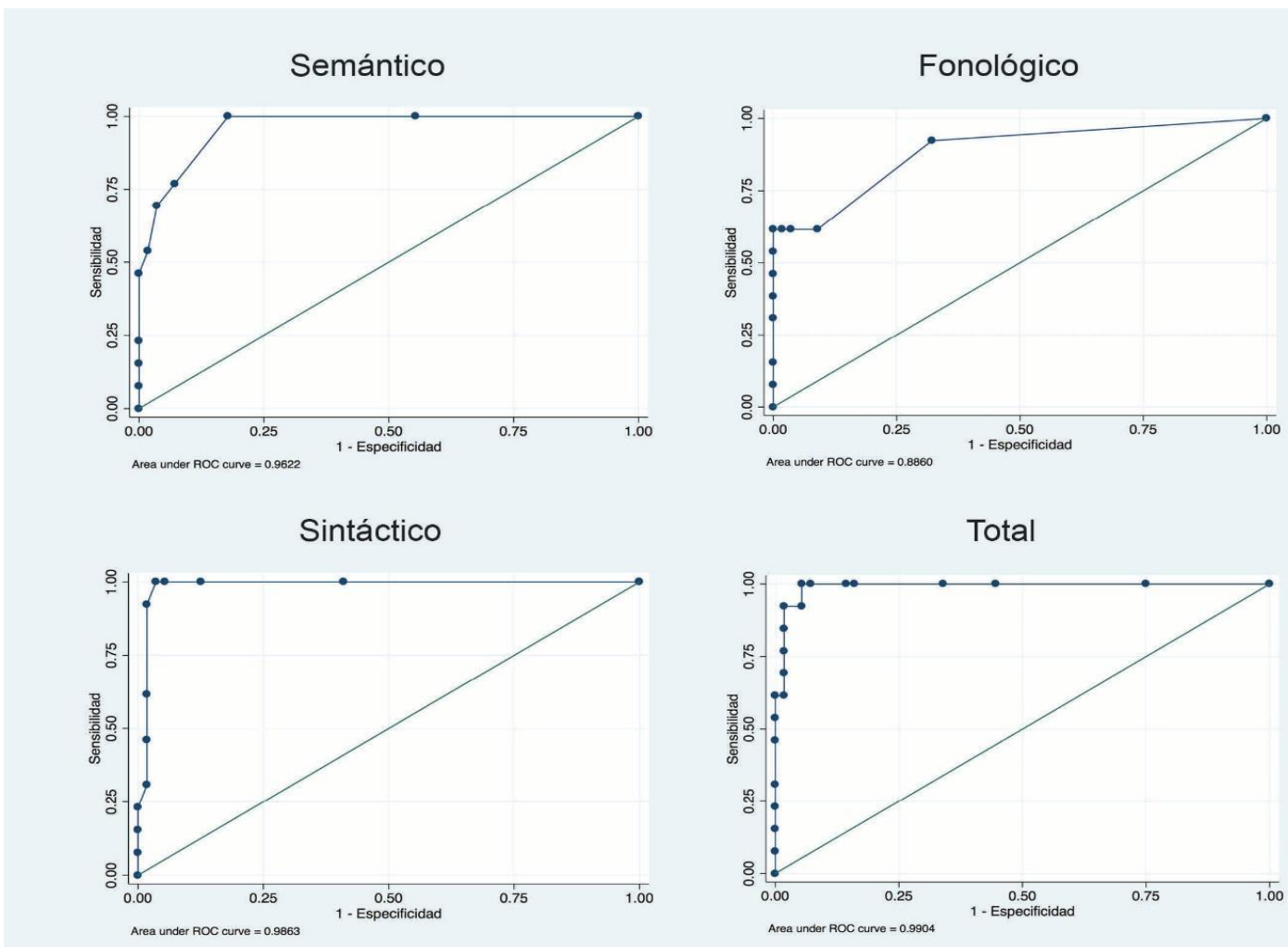

Figura 1. Curvas ROC y sus respectivas áreas para el puntaje global del test ScreeLing y sus subpruebas.

Respecto a las subpruebas, el puntaje de corte obtenido en la subprueba semántica fue de 22 puntos, con una sensibilidad de $92,86 \%$, especificidad del $76,92 \%$ y área bajo la curva ROC de 0,96 . Con un puntaje de corte de 21 puntos en la subprueba fonológica se obtiene una sensibilidad del 100\%, especificidad de $61.54 \%$ y un área bajo la curva ROC de 0.87. Finalmente, en la subprueba sintáctica con un puntaje de corte de 21 puntos, la sensibilidad es de $96,43 \%$, la especificidad del $100 \%$ y el área bajo la curva ROC de 0.99 . Ver tabla 4.
Tabla 4. Puntajes de corte, sensibilidad y especificidad para el Test ScreeLing y sus subpruebas.

\begin{tabular}{lcccc}
\hline $\begin{array}{l}\text { ScreeLing } \\
\text { (puntaje) }\end{array}$ & $\begin{array}{c}\text { Puntaje } \\
\text { de corte }\end{array}$ & $\begin{array}{c}\text { Sensibilidad } \\
(\%)\end{array}$ & $\begin{array}{c}\text { Especificidad } \\
(\%)\end{array}$ & $\begin{array}{c}\text { Clasificados } \\
\text { correctamente } \\
(\%)\end{array}$ \\
\hline $\begin{array}{l}\text { Subprueba } \\
\text { semántica }\end{array}$ & 22 & 92,86 & 76,92 & 89,86 \\
$\begin{array}{l}\text { Subprueba } \\
\text { fonológica }\end{array}$ & 21 & 100 & 61,54 & 92,75 \\
$\begin{array}{l}\text { Subprueba } \\
\text { sintáctica }\end{array}$ & 21 & 96,43 & 100 & 97,1 \\
$\begin{array}{l}\text { Global } \\
\text { ScreeLing }\end{array}$ & 67 & 94,64 & 100 & 95,65 \\
\hline
\end{tabular}




\section{DISCUSIÓN}

El objetivo de este estudio fue analizar la sensibilidad y especificidad de la versión en español del test ScreeLing en una muestra de población chilena en etapa aguda post ACV. Dicho test ha sido descrito en Holanda como una herramienta válida y confiable para el diagnóstico de afasia en usuarios en etapa aguda post ACV (El Hachioui et al., 2012). El test ScreeLing permitió diferenciar entre el grupo de adultos neurológicamente sanos y los usuarios con diagnóstico de afasia producto de ACV. Además, el instrumento mostró una alta especificidad y sensibilidad.

En relación con el puntaje global del test, el corte del presente estudio fue 67 puntos, siendo similar al obtenido en Holanda. El nivel de sensibilidad y especificidad es similar a los reportados por el estudio preliminar del test Screeling por Doesborgh et al. (2003) (86\% y $96 \%$ respectivamente) y el estudio final de validación de El Hachioui et al. (2012) (94\% y $81 \%$ respectivamente). En este trabajo, el puntaje de corte, se decidió por un balance entre sensibilidad y especificidad, pero sin sacrificar la sensibilidad, mientras que en la versión final holandesa de El Hachioui et al. (2012) se privilegió la sensibilidad. Esto quiere decir que el test ScreeLing es un instrumento válido para diferenciar entre sujetos sanos y quienes presentan déficits lingüísticos asociados a ACV.

Al comparar la presente investigación con el estudio de validación original, se puede observar que el promedio total es similar entre controles de ambas muestras. En cambio, el promedio del total entre los casos de la presente muestra y del estudio holandés difieren por aproximadamente 4 puntos. Esto se puede asociar a las diferencias en cuanto al nivel educacional de la muestra de ambos países. En Chile un 64,9\% de personas, entre 25 y 64 años de edad, están graduadas de por lo menos educación media superior. En cambio, en Holanda aumenta en más de 10 puntos este porcentaje, ya que corresponde al $78,4 \%$, de personas (OECD, 2019). Futuras investigaciones con un tamaño muestral mayor deberían estudiar el efecto del nivel educativo en las puntuaciones del test.

Al comparar los resultados obtenidos en el presente estudio con aquellos de la investigación que lo validó originalmente (El Hachioui et al., 2012), aparecen ciertas discrepancias en la magnitud de las diferencias entre grupos en las puntuaciones de algunas subpruebas del test. Por ejemplo, la mayor diferencia en la muestra del estudio Holandés correspondió al nivel fonológico, mientras que en el presente estudio la diferencia con mayor magnitud se observó en la subprueba sintáctica. Además, existirían diferencias al comparar las puntuaciones del grupo de casos entre ambos estudios para el nivel fonológico. Las anteriores diferencias podrían ser atribuidas al tipo de afasia que conformó la muestra de casos en ambos estudios, por lo que una futura investigación debería considerar dicha variable.

\section{Limitaciones}

En el estudio se advierten dos limitaciones relevantes. Una relacionada con el tamaño de la muestra y la otra, con el rendimiento del grupo control en el test.

La muestra evaluada fue pequeña, con sólo 13 casos, los cuales fueron seleccionados por conveniencia, no siendo representativa de la población chilena. Sin embargo, el test permite diferenciar con tal precisión a los pacientes afásicos de aquellos sanos que el tamaño muestral fue adecuado para realizar comparaciones entre grupos. Debido al reducido tamaño muestral tampoco se realizó un análisis de confiabilidad obteniendo medidas como un Alfa de Cronbach. No se efectuaron mayores ajustes al modelo de regresión (considerando variables tales como edad y años de educación) siguiendo las recomendaciones de Vittinghoff \& McCulloch (2007). Este estudio sugiere que se debe tener al menos 30 casos para elaborar modelos logísticos multivariado.

Respecto al rendimiento del grupo control, se observó efecto techo en la mediana y en la desviación estándar en el puntaje total del test. Es decir, la mayoría de los sujetos sin déficits lingüísticos no presentan dificultades en la realización de las tareas del test ScreeLing. Lo anterior significaría que el test es adecuado para diferenciar los déficits asociados a la Afasia y no las variaciones propias del envejecimiento o del nivel de escolaridad.

\section{Proyecciones}

Además de incluir una muestra mayor y más representativa de pacientes, futuras investigaciones podrían evaluar el efecto de variables como localización de la lesión, diagnóstico previo de otras enfermedades y antecedentes de ACV previos. Otro aspecto a tratar a futuro sería analizar la especificidad de este test para el diagnóstico diferencial de afasia frente a otras alteraciones del lenguaje, como son los trastornos cognitivos comunicativos por diversas etiologías (demencias, traumatismo encéfalo craneano, entre otros).

También, se propone explorar una comparación entre el rendimiento en fase aguda y crónica del ACV, para analizar los cambios en el desempeño entre ambas etapas y reflexionar sobre si la terapia fonoaudiológica efectuada es efectiva de manera cualitativa y cuantitativa. 
Finalmente, la validación final de este instrumento debe incluir mayor muestra para evaluar de mejor forma el efecto de la edad y del nivel educacional en las puntuaciones de las subpruebas del test. Lo anterior, dado que es necesario estratificar por rangos de edad y educación para estudiar el efecto de dicha variable.

\section{CONCLUSIONES}

El test ScreeLing es un test práctico, de rápida aplicación, que se podría convertir en un instrumento que contribuiría no sólo en la pesquisa de afasia, sino en la descripción de los déficits lingüísticos asociados a ésta. Al dar información de manera precoz, el test permite iniciar una intervención temprana, dando prioridad a los componentes de lenguaje más afectados.

\section{AGRADECIMIENTOS}

Los autores agradecen a los colaboradores en el proceso de inclusión de pacientes, a Flga. Bernardita Letelier, Flga. Josette Sáez, Flga. Elena Medina del Servicio de Neurología del Hospital Dr. Sótero del Río. Tambien al Flgo. Nelson Saá y Patricia Feliu del Servicio de Neurología del Centro de Salud UC-Christus.

\section{REFERENCIAS}

Adams, H. P., Davis, P. H., Leira, E. C., Chang, K. C., Bendixen, B. H., Clarke, W. R., Woolson, R. F., \& Hansen, M. D. (1999). Baseline NIH Stroke Scale score strongly predicts outcome after stroke: A report of the Trial of Org 10172 in Acute Stroke Treatment (TOAST). Neurology, 53(1), 126-131. https://doi.org/10.1212/wnl.53.1.126

De Renzi, E., \& Faglioni, P. (1978). Normative data and screening power of a shortened version of the Token Test. Cortex; a Journal Devoted to the Study of the Nervous System and Behavior, 14(1), 41-49. https://doi.org/10.1016/s00109452(78)80006-9

Doesborgh, S. J. C., van de Sandt-Koenderman, W. M. E., Dippel, D. W. J., van Harskamp, F., Koudstaal, P. J., \& Visch-Brink, E. G. (2003). Linguistic deficits in the acute phase of stroke. Journal of Neurology, 250(8), 977-982. https://doi.org/10.1007/s00415-003-1134-9
El Hachioui, H., Sandt-Koenderman, M. W. M. E., Dippel, D. W. J., Koudstaal, P. J., \& Visch-Brink, E. G. (2012). The ScreeLing: Occurrence of linguistic deficits in acute aphasia post-stroke. Journal of Rehabilitation Medicine, 44(5), 429-435. https://doi.org/10.2340/16501977-0955

El Hachioui, H., Visch-Brink, E. G., de Lau, L. M. L., van de Sandt-Koenderman, M. W. M. E., Nouwens, F., Koudstaal, P. J., \& Dippel, D. W. J. (2017). Screening tests for aphasia in patients with stroke: A systematic review. Journal of Neurology, 264(2), 211-220. https://doi.org/10.1007/s00415-016-8170-8

Flamand-Roze, C., Falissard, B., Roze, E., Maintigneux, L., Beziz, J., Chacon, A., Join-Lambert, C., Adams, D., \& Denier, C. (2011). Validation of a new language screening tool for patients with acute stroke: The Language Screening Test $\begin{array}{llll}\text { (LAST). } & \text { Stroke, } & \text { 1224-1229. }\end{array}$ https://doi.org/10.1161/STROKEAHA.110.609503

González, F., Lavados, P., \& Olavarría, V. (2017). Incidencia poblacional, características epidemiológicas y desenlace funcional de pacientes con ataque cerebrovascular isquémico y afasia. Revista médica de Chile, 145(2), 194-200. https://doi.org/10.4067/S0034-98872017000200007

Goodglass, H., Kaplan, E., \& Barresi, B. (2005). Test de Boston para el diagnóstico de la afasia: Adaptación española. http://bibliotecadigital.usb.edu.co/bitstream/10819/275/2/Interpretacion_Expresi ones_Metaforicas_Camacho_2011_Anexo1.pdf

Laska, A. C., Bartfai, A., Hellblom, A., Murray, V., \& Kahan, T. (2007). Clinical and prognostic properties of standardized and functional aphasia assessments. Journal of Rehabilitation Medicine, 39(5), 387-392. https://doi.org/10.2340/16501977-0070

Ministerio de Salud Chile. (2013). Guía Clínica Accidente Cerebro Vascular Isquémico en personas de 15 años y más. MINSAL. http://web.minsal.cl/portal/url/item/7222754637e58646e04001011f014e64.pdf

Nouwens, F., Jong-Hagelstein, M. de, Lau, L. M. L. de, Dippel, D. W. J., Koudstaal, P. J., Sandt-Koenderman, W. M. E. van de, \& Visch-Brink, E. G. (2014). Severity of aphasia and recovery after treatment in patients with stroke. Aphasiology, 28(10), 1168-1177. https://doi.org/10.1080/02687038.2014.907865

OECD. (2019). Educación. Better Life Index. Educación. http://www.oecdbetterlifeindex.org/es/topics/education-es/

Salter, K., Jutai, J., Foley, N., Hellings, C., \& Teasell, R. (2006). Identification of aphasia post stroke: A review of screening assessment tools. Brain Injury, 20(6), 559-568. https://doi.org/10.1080/02699050600744087

Vittinghoff, E., \& McCulloch, C. E. (2007). Relaxing the Rule of Ten Events per Variable in Logistic and Cox Regression. American Journal of Epidemiology, 165(6), 710-718. https://doi.org/10.1093/aje/kwk052 